\title{
Progressive Image Transmission Scheme in Wireless Multimedia Sensor Networks
}

\author{
Enyan Sun ${ }^{1,2}$, Xuanjing Shen ${ }^{1}$, Haipeng Chen ${ }^{1}$ and Chuanyun Wang ${ }^{2}$ \\ ${ }^{1}$ College of Computer Science and Technology, Jilin University, Changchun, \\ China \\ ${ }^{2}$ School of Computer Science, Shenyang Aerospace University, Shenyang, China \\ sunenyan418418@163.com
}

\begin{abstract}
Wireless Multimedia Sensor Networks which are the higher version of Wireless Sensor Networks have broader application domains. However, a large number of images' transmission can consume a lot of energy of sensor nodes in Wireless Multimedia Sensor Networks. Considering the tradeoff between the energy consumption and image quality, Progressive Image Transmission Scheme (PITS) is proposed. PITS can meet the demand of image quality of the sink. Meanwhile, PITS can reduce the image data which is transmitted to the sink and furthermore reduce the energy consumption of sensor nodes. Simulation verifies the feasibility of PITS in Wireless Multimedia Sensor Networks.
\end{abstract}

Keywords: Wireless Multimedia Sensor Networks, Image Transmission, Image Compression, Discrete Cosine Transform

\section{Introduction}

Wireless Multimedia Sensor Networks (WMSNs) are the high version of Wireless Sensor Networks (WSN). The inexpensive CMOS cameras and microphones have fostered the development of WMSNs. WMSNs can retrieve video streams and audio streams, still images and scalar sensor data from the surrounding environment. WMSNs will have more application domains such as multimedia surveillance sensor networks, telemedicine sensor networks, environment monitoring etc [1-3].

Due to limited energy and computation capability of sensor nodes, it is difficult to transmit video streams in Wireless Multimedia Sensor Networks. Now still images are mainly data which are processed in Wireless Multimedia Sensor Networks. Sensor nodes have limited energy and the transmission of raw images needs more energy. So images will be compressed before the transmission. Image compression can remove the redundant information from the raw image [4, 5]. JPEG2000 have good performance such as progressive transmission, meanwhile, JPEG2000 is very complex and cannot be applied in tiny sensor nodes.

JPEG which is based on Discrete Cosine Transform is simpler than JPEG2000. JPEG is more suitable for Wireless Sensor Multimedia Networks. However, JPEG lacks progressive transmission capability. Progressive Image Transmission Scheme (PITS) which is based upon 2-Dimension Discrete Cosine Transform is proposed in the paper. PITS can decrease energy consumption sensor nodes and be more suitable for WMSNs.

The paper is organized as follows. In Section 2, we present the related works about the image compression and transmission in wireless multimedia sensor networks. In Section 3, 2-D Discrete Cosine Transformation is introduced. Progressive Image Transmission Scheme is proposed in Section 4. Section 5 includes the experiments and results. Finally, our conclusion and future research direction are included in Section 6. 


\section{Related Works}

Hadi S. Aghdasi et al. [6] proposed a thorough architecture for video transmission over Wireless Video Sensor Networks WVSN. The model of compressing and transmitting video is built in the application layer in WVSN. The proposed model is called as MMPEG which is based on MPEG-2 standard. The M-MPEG utilizes Main-Frame (MFrame) and Difference-Frame (D-Frame). Considering energy saving and video quality, different image blocks are given different priorities in the M-Frame and D-Frame. This architecture has better performance than other conventional architectures.

Abdelhamid Mammeri et al. [7] proposed S-JPEG which adapts JPEG to the energy requirement of Visual Sensor Networks (VSN). Reduced Block Size (RBS) consists in upper-left squared portion of each block of $8 * 8$ DCT coefficients of the image. Only RBS is selected to save energy and other DCT coefficients are ignored in the image compression. Compare to JPEG, S-JPEG can save the sensor nodes' energy and prolong the network lifetime.

Leila Makkaoui et al. [8] proposed Fast Zonal DCT-based image compression for Wireless Camera Sensor Networks. The fast zonal DCT-based image compression uses the square and triangle approaches to reach the trade-off between energy consumption and image distortion. The fast zonal DCT-based image compression can decrease energy consumption of the senor nodes.

S.A.Hussain et al. [9] proposed energy efficient image compression in wireless sensor networks. Image Subtraction with Quantization of image (ISQ) is proposed to reduce the communication latency and energy consumption. ISQ transmits only the changes in the image to the sink instead of sending back the whole image.

We [10] proposed a novel Low Energy Image Compression Algorithm (LEICA) that is based on an interest area in WMSNs. LEICA is based on the idea that ROI is compressed at a low compression ratio and tiles are compressed at a high compression ratio. LEICA can increase the compression ratio of the whole image while meeting an image quality of ROI. LEICA can decrease the energy consumption on the computation and transmission. Compared to S-JPEG [7] and JPEG, LEICA can prolong the network lifetime.

Syed Mahfuzul Aziz et al. [11] proposed Energy Efficient Image Transmission in Wireless Multimedia Sensor Networks. The object extraction architecture is used to reduce the energy consumption of image transmission. Only one node transmits data at a time to reduce collision, congestion and retransmission.

Considering that JPEG2000 is also high complexity and cannot be realized in an individual senor node which is limited in memory capacity, computation power and energy, Distributed image compression and transmission scheme (DICT) [12] is proposed to overcome the problem of scarce resources of the individual sensor node and uneven energy consumption. DICT is proposed to lessen the individual sensor node's burden of computation, memery and energy. DICT can balance the energy consumption of sensor nodes in networks and extend the network lifetime. Simulation experiment shows that DICT is better than centralized image compression and transmission scheme such as CCIS and CHIS in the energy consumption balance and the network lifetime.

\section{Theory of Discrete Cosine Transform}

The 2-Dimensional Discrete Cosine Transform (2-D DCT) is the core part of JPEG. 2D DCT can transform an image from the spatial domain to the frequency domain. Generally the raw image is divided into small blocks and each block consists of $8 \times 8$ pixels. Each block is transformed from the spatial domain to the frequency domain by the 2-D DCT. The 2-D DCT is expressed as Equation (1).

$$
F(u, v)=\frac{1}{4} C(u) C(v) \sum_{x=0}^{k-1} \sum_{y=0}^{k-1} f(x, y) \cos \left(\frac{(2 x+1) u \pi}{2 k}\right) \cos \left(\frac{(2 y+1) v \pi}{2 k}\right)
$$


where

$$
C(z)=\left\{\begin{array}{c}
1 / \sqrt{2} \text { if } z=0 \\
1 \text { otherwise }
\end{array}\right.
$$

The 2-D DCT is also expressed as Equation (3).

$$
F(k \times k)=A(k \times k) P(k \times k) A^{T}(k \times k)
$$

The value of k is $8 . P(k \times k)$ is a raw pixel matrix. $A(k \times k)$ is a discrete cosine matrix. $F(k \times k)$ is a DCT coefficient matrix. The transformed values of each block make up a DCT coefficient matrix which consists of $8 \times 8$ elements.

\section{Progressive Image Transmission Scheme}

Figure 1 shows the image transmission path in Wireless Multimedia Sensor Networks. Path 1 is traditional transmission path. Sensor nodes which are near the event firstly capture raw images. Then, these raw images are compressed by the sensor nodes. The compressed images are transmitted to the sink by multiple relay sensor nodes.

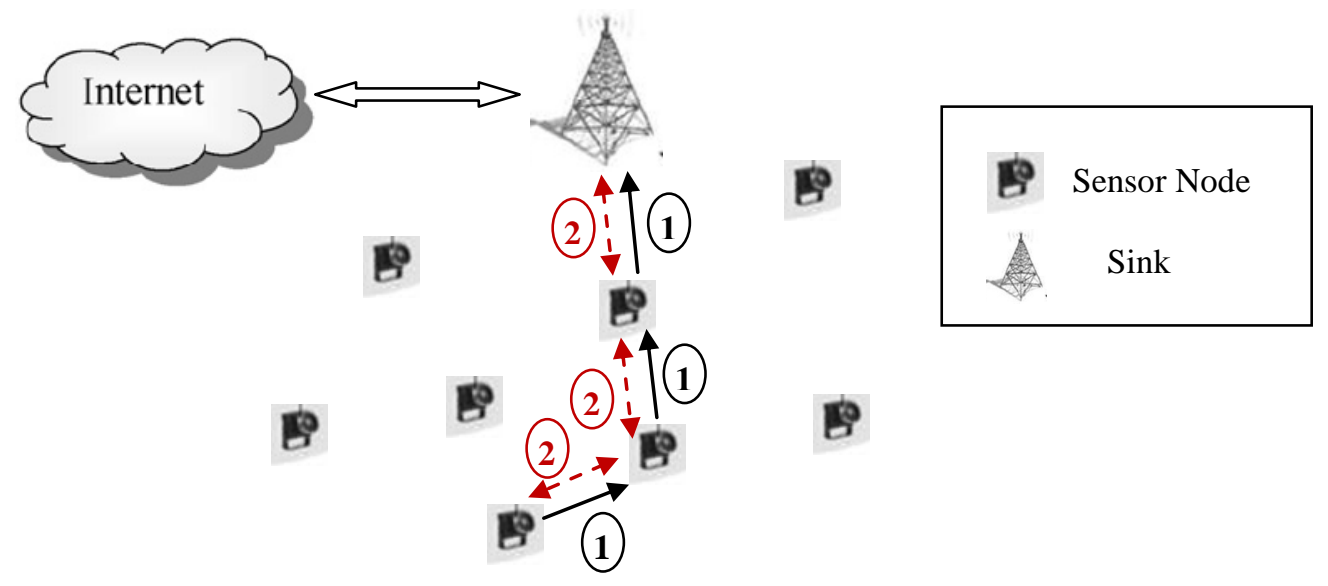

Figure 1. Image Transmission Path Diagram

The above image transmission scheme has obvious disadvantages. Image quality of those images that the sink has received cannot conform to the actual demand on occasion. The sensor node compresses images at higher compression ratio, and it leads to poor image quality. When the sink needs good quality images, these poor quality images cannot reach the demand.

If the sensor node compressed images at low compression ratio, and the compressed image quality is good. When transmitting these images to the sink, relay sensor nodes will consume a lot of energy.

Table 1 shows data value of a block of the raw image which consists of $8^{*} 8$ pixels. The image block can be transformed by 2-D DCT through Equation (1). Table 2 shows the transformed DCT coefficient matrix.

Table 1. A Block of the Raw Image

\begin{tabular}{|l|l|l|l|l|l|l|l|}
\hline 162 & 162 & 162 & 161 & 162 & 157 & 163 & 161 \\
\hline 162 & 162 & 162 & 161 & 162 & 157 & 163 & 161 \\
\hline 162 & 162 & 162 & 161 & 162 & 157 & 163 & 161 \\
\hline 162 & 162 & 162 & 161 & 162 & 157 & 163 & 161 \\
\hline 162 & 162 & 162 & 161 & 162 & 157 & 163 & 161 \\
\hline 164 & 164 & 158 & 155 & 161 & 159 & 159 & 160 \\
\hline 160 & 160 & 163 & 158 & 160 & 162 & 159 & 156 \\
\hline 159 & 159 & 155 & 157 & 158 & 159 & 156 & 157 \\
\hline
\end{tabular}


Table 2. DCT Coefficient Matrix

\begin{tabular}{|c|c|c|c|c|c|c|c|}
\hline 1284 & 4.7683 & 3.24 & -0.2 & 0.25 & -0.55389 & -4.5894 & 5.6385 \\
\hline 7.947 & -0.788 & 0.555 & -4.93 & 1.9602 & 2.9784 & -3.7971 & 3.3222 \\
\hline-5.03 & -0.297 & -1.55 & 1.725 & -0.6765 & -0.4525 & 1.8499 & -2.2002 \\
\hline 2.262 & 1.139 & 1.704 & 0.924 & -0.76061 & -1.3686 & 0.21428 & 1.1422 \\
\hline-1 & -1.15 & -0.34 & -1.36 & 1.75 & 1.1189 & -1.4815 & -0.67289 \\
\hline 1.211 & 0.4561 & -1.8 & -0.11 & -2.0116 & 0.70966 & 1.6866 & 0.75515 \\
\hline-1.7 & 0.265 & 3.1 & 1.636 & 1.6332 & -2.2546 & -1.1982 & -0.90111 \\
\hline 1.326 & -0.415 & -2.38 & -1.59 & -0.88457 & 1.9693 & 0.55319 & 0.65399 \\
\hline
\end{tabular}

Table 3. Zigzag Arrangement of DCT Coefficients

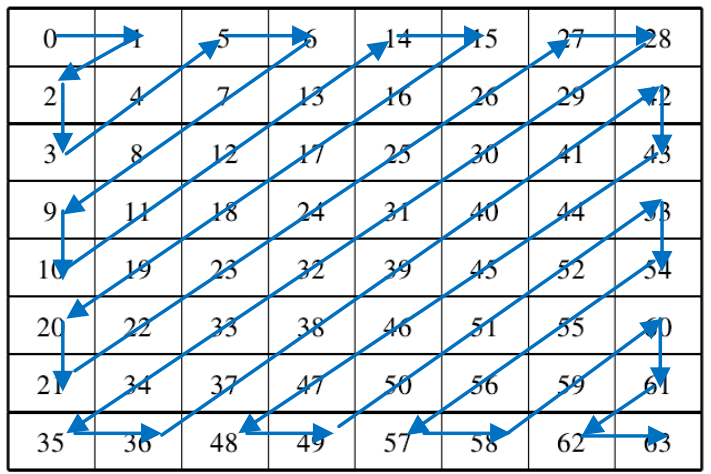

The most upper left data whose value is 1284 is the DC coefficient in Table 2. The DC coefficient monopolizes most energy of the image and has most effect on the quality of an image. The upper left low and middle AC coefficients occupy some energy of an image and have a little effect on the quality of an image. Lower right high AC coefficients which occupy less energy of an image have less effect on the quality of an image. Table 3 shows zigzag arrangement according the importance of DCT coefficients.

Lena which is $512 * 512$ pixels is selected as the test image. Figure 2-7 shows the result when selecting different number of DCT coefficients.

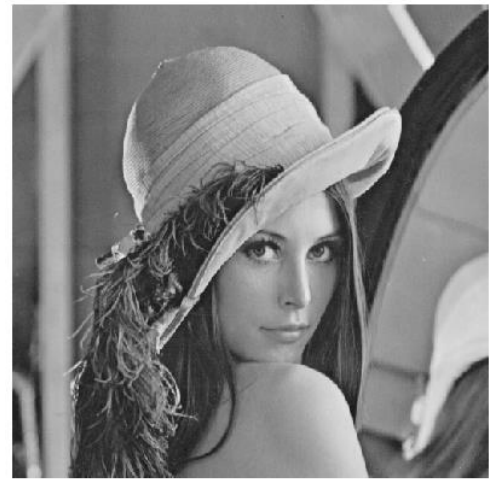

Figure 2. Raw Image

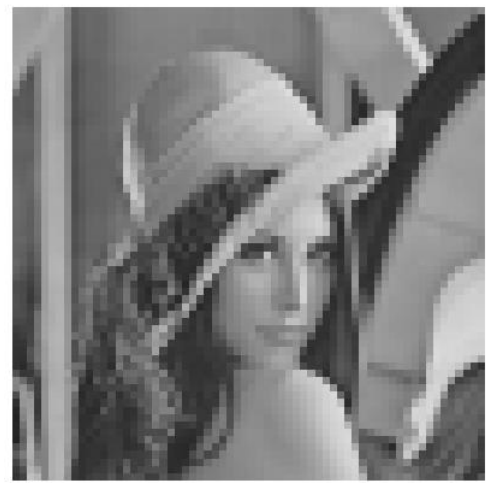

Figure 3. DC and 0 AC 


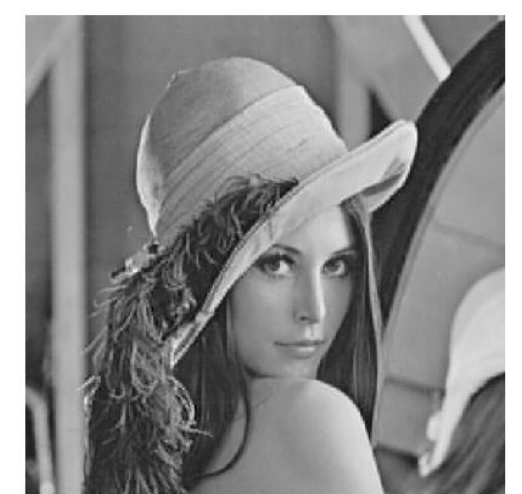

Figure 6. DC and 9 AC

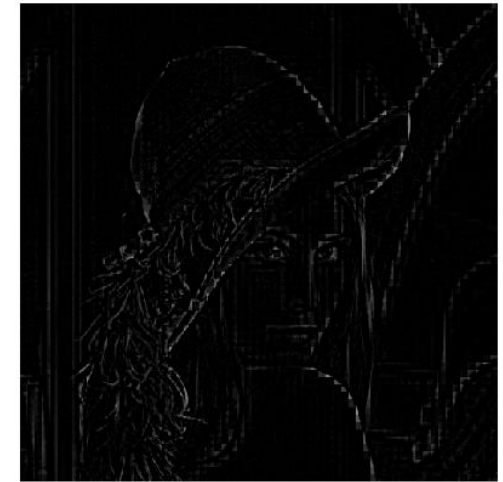

Figure 7. No DC and $63 \mathrm{AC}$

Figure 3 shows the image when selecting DC coefficient. There are obviously block effect on the image. When selecting DC coefficient and one AC coefficient, there are yet block effect on the image as Figure 4. Generally, we can endure the quality of an image of Figure 5. More number of AC coefficients has little effect on the quality of an image. When selecting 63 AC coefficients and no DC coefficient, Figure 7 shows the results and it is bad results. 


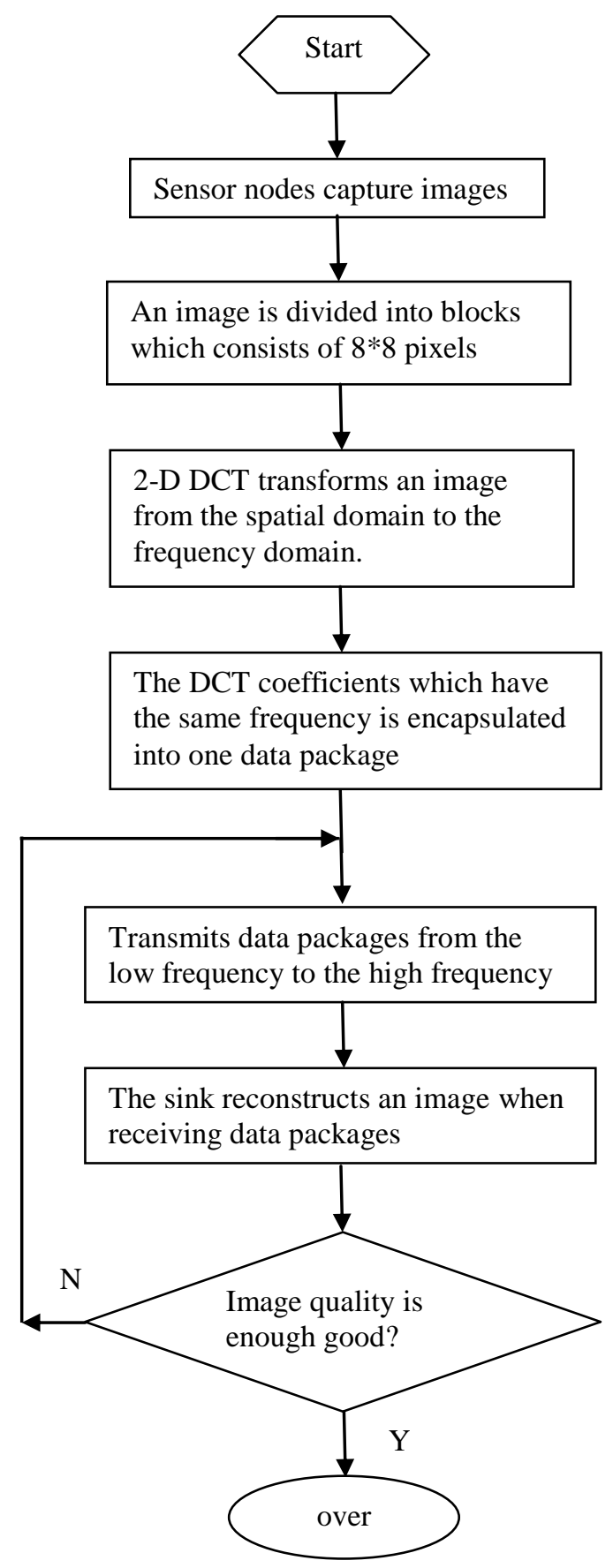

Figure 8. Flowchart of Progressive Image Transmission Scheme

So the idea of Progressive Image Transmission Scheme (PITS) is that the sensor node transmits DCT coefficients to the sink according to zigzag arrangement sequence of Table 3. The transmission path is described Path 2 as Figure 1. Once the sink receives image data, the sink rebuilds the image. When the quality of the rebuilt image may satisfy the actual demand, the sink gives out the 'stop' demand to the sensor nodes.

Progressive Image Transmission Scheme (PITS) is described as Figure 8. The detail procedure is described as the following steps.

(1) When a monitored object appears in wireless sensor network, the source sensor nodes which are waken up continuously capture the raw images.

(2) An image can be divided into a number of blocks. A block consists of $8 * 8$ pixels. 
(3) Each block can be calculated by 2-D DCT which can transform an image from the spatial domain to the frequency domain, which can generate DCT coefficient matrixes.

(4) The DCT coefficients which have the same frequency are encapsulated into one data package.

(5) The sensor node transmits data packages from the low frequency to the high frequency according to Zigzag arrangement of DCT coefficients of Table 3.

6) The sink reconstructs an image when receiving data packages using 2-D IDCT. If the quality of the images have meet the demand of the application, the sink notifies the source node to stop transmit data packets. If the quality of the images cannot meet the demand of the application, the sink notifies the source node to continue to transmit data packets of images.

\section{Simulation and Analysis}

Energy consumption is a fundamental concern in Wireless Multimedia Sensor Networks. Energy of sensor nodes is limited and cannot be recharged. So the simulation about energy consumption is done. In wireless multimedia sensor networks, the node A transmits $\mathrm{k}$ bits to the node $\mathrm{B}$ and the distance between two nodes is $\mathrm{d}$. The energy that the node A consumes is computed by Equation (4). The energy that the node B consumes is computed by Equation (5).

$$
\begin{aligned}
& E_{T X}(k, d)=\left\{\begin{array}{l}
k \bullet E_{\text {elec }}+k \bullet \varepsilon_{f s} \bullet d^{2}, d<d_{0} \\
k \bullet E_{\text {elec }}+k \bullet \varepsilon_{\text {amp }} \bullet d^{4}, d \geq d_{0}
\end{array}\right. \\
& E_{R X}(k)=k \bullet E_{\text {elec }}
\end{aligned}
$$

Eelec is the energy consumption of the electric circuit when the node transmits or receives data. $\varepsilon_{f s}$ is $10 \mathrm{pJ} / \mathrm{bitm} 2$, and $\varepsilon_{a m p}$ is $0.0013 \mathrm{pJ} / \mathrm{bit} \mathrm{m}^{4} . \mathrm{d}_{0}$ is $87 \mathrm{~m}$.

Figure 9 shows energy consumption of sensor nodes with the increasing distance. Obviously, more number of AC coefficients which are joined in the image lead to more energy consumption. PITS can decrease energy consumption of the sensor nodes through decreasing $\mathrm{AC}$ coefficients according to the quality of images.

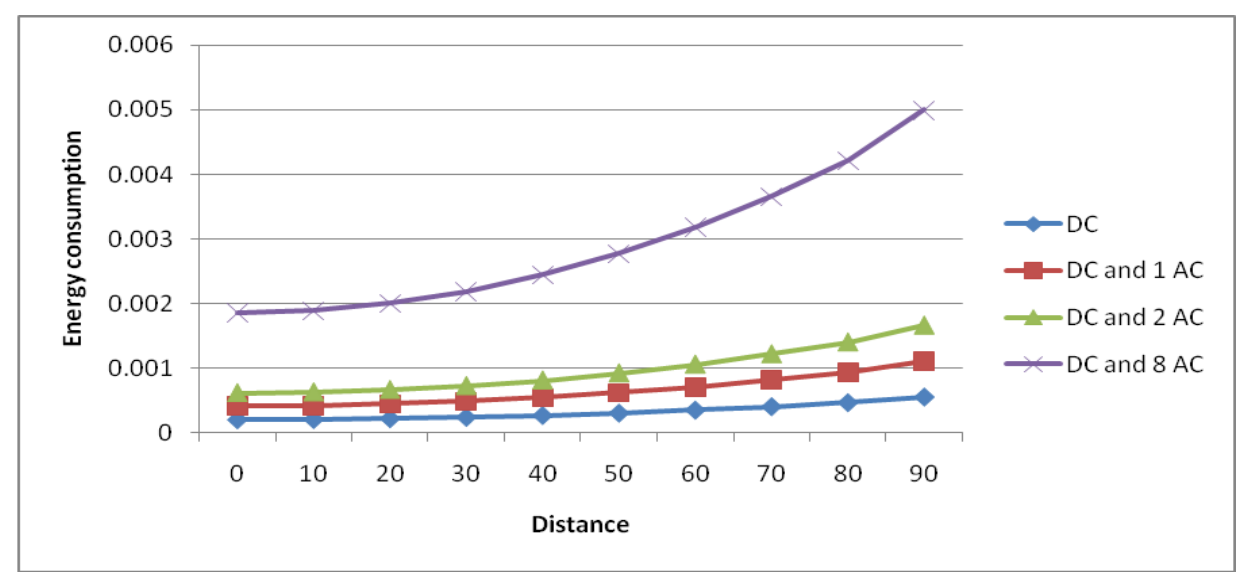

Figure 9. Energy Consumption of Sensor Nodes 


\section{Conclusion and Future}

Wireless multimedia sensor nodes are limited in energy. A lot of still images are transmitted in Wireless Multimedia Sensor Networks. However, images' transmission can consume lots of energy of wireless multimedia sensor nodes in wireless multimedia sensor networks. JPEG2000 is too complex to suitable for Wireless Multimedia Sensor Nodes. JPEG cannot realize progressive image transmission. PITS which is proposed to meet the need of Wireless Multimedia Sensor Nodes can adjust the number of image data according to demand of image quality of the sink. PITS can realize progressive image transmission from low frequency coefficients to high frequency coefficients. PITS can dramatically decrease energy consumption of wireless multimedia sensor nodes through decreasing high frequency DCT coefficients while meeting the image quality of Wireless Multimedia Sensor Node. PITS is suitable for Wireless Multimedia Sensor Networks. In the future, PITS will furthermore be studied to apply to smart monitoring systems in wireless multimedia sensor networks.

\section{Acknowledgements}

This work was supported by Educational Department of Liaoning Province of China un der Grant No.L2013067.

\section{References}

[1] I.F. Akyildiz, T. Melodia and K. R. Chowdhury, "A survey on wireless multimedia sensor networks", Computer networks, vol. 51, no. 4, (2007), pp. 921-960.

[2] S. Misra, M. Reisslein and G. Xue, "A survey of multimedia streaming in wireless sensor networks", Communications Surveys \& Tutorials, IEEE, vol. 10, no. 4, (2008), pp. 18-39.

[3] I.T. Almalkawi, M. Guerrero Zapata, J. N. Al-Karaki and J. Morillo-Pozo, "Wireless multimedia sensor networks: current trends and future directions", Sensors (Basel, Switzerland), vol. 10, no. 7, (2010), pp. 6662.

[4] Chew, W. Li, L.-M. Ang and K. P. Seng, "Survey of image compression algorithms in wireless sensor networks", In Information Technology, 2008. ITSim 2008. International Symposium on, IEEE, vol. 4, (2008), pp. 1-9.

[5] A. Mammeri, B. Hadjou and A. Khoumsi, "A Survey of Image Compression Algorithms for Visual Sensor Networks", ISRN Sensor Networks 2012, (2012).

[6] H.S. Aghdasi, M. Abbaspour, M. Ebrahimi Moghadam and Y. Samei, "An energy-efficient and highquality video transmission architecture in wireless video-based sensor networks", Sensors, vol. 8, no. 8, (2008), pp. 4529-4559.

[7] A. Mammeri, A. Khoumsi, D. Ziou and B. Hadjou, "Energy-aware JPEG for visual sensor networks", In Proceedings of The 2008 Maghrebian Conference on Software Engineering and Artificial Intelligence, IEEE, (2008), pp. 1-7.

[8] L. Makkaoui, V. Lecuire and J-M. Moureaux, "Fast zonal DCT-based image compression for wireless camera sensor networks", In Image Processing Theory Tools and Applications (IPTA), 2010 2nd International Conference on, IEEE, (2010), pp. 126-129.

[9] S. A. Hussain, M. I. Razzak, A. A. Minhas, M. Sher and G. R. Tahir, "Energy efficient image compression in wireless sensor networks", Aceee International Journal on signal \& Image processing, vol. 1, no. 2, (2010).

[10] E. Sun, X. Shen and H. Chen, "A Low Energy Image Compression and Transmission in Wireless Multimedia Sensor Networks", Procedia Engineering, vol. 15, (2011), pp. 3604-3610.

[11] S. Aziz and D. Pham, "Energy Efficient Image Transmission in Wireless Multimedia Sensor Networks", (2013), pp. 1-4.

[12] E. Sun, X. Shen, H. Chen and C. Wang, "Distributed Image Compression and Transmission Scheme in Wireless Multimedia Sensor Networks”, TELKOMNIKA Indonesian Journal of Electrical Engineering, vol. 12, no. 1, (2014).

[13] M. V. Petkov, K. Obraczka and R. Manduchi, "Characterizing energy consumption in a visual sensor net work testbed", In 2nd International IEEE/Create-Net Conference on Testbeds and Research Infrastructures for the Development of Networks and Communities, (2006). 
Authors

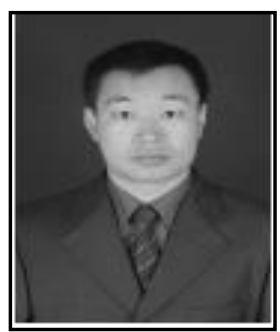

Enyan Sun, he received the B.S. degree in Computer Science and Technology from the College of Computer and Science and Technology, Jilin University, China, in 2002 and received the M.S. degree in Computer Application Technology from the school of Computer Science, Shenyang Aerospace University, China, in 2007. He is now a PH.D. candidate at the College of Computer and Science and Technology, Jilin University. He has worked as a teacher in the College of Computer Science of Shenyang Aerospace University from 2002. His major research interests include the routing protocols and image compression in wireless multimedia sensor networks,

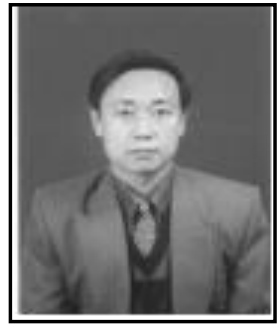

Xuanjing Shen, he is a professor at the College of Computer Science and Technology, Jilin University. He received his Ph.D. degree from Harbin Institute of Technology in 1990. His research interests cover wireless multimedia sensor networks, computer image processing, intelligent measurement system, and optical-electronic hybrid system.

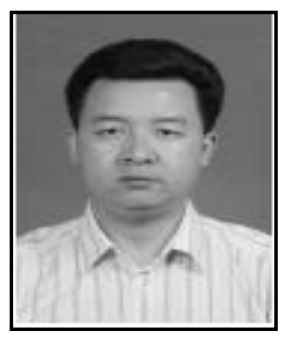

Haipeng Chen, he is a Ph.D. and associate professor at the College of Computer Science and Technology, Jilin University. His research interests cover image processing, pattern recognition and wireless multimedia sensor networks.

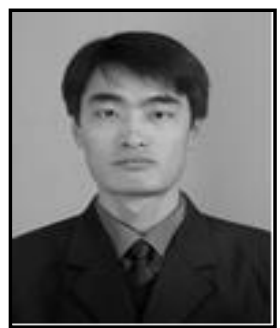

Chuanyun Wang, he received the M.A. degree in technology of computer application from Shenyang Aerospace University, China, in 2009. Then he joined the College of Computer Science of Shenyang Aerospace University, and is a lecturer at present. His current research interests include wireless sensor networks and internet of things. 
International Journal of Control and Automation

Vol. 10, No. 1 (2017) 